\title{
OPEN Changes on proteomic and metabolomic profile in serum of mice induced by chronic exposure to tramadol
}

Shukun Jiang ${ }^{1,4}$, Guojie Liu ${ }^{2,4}$, Huiya Yuan ${ }^{1}$, Enyu Xu ${ }^{1}$, Wei Xia ${ }^{1}$, Xiaoyu Zhang ${ }^{3}$, Junting Liu ${ }^{1}$ \& Lina Gao ${ }^{1 \bowtie}$

Tramadol is an opioid used as an analgesic for treating moderate or severe pain. The long-term use of tramadol can induce several adverse effects. The toxicological mechanism of tramadol abuse is unclear. Limited literature available indicates the change of proteomic profile after chronic exposure to tramadol. In this study, we analyzed the proteomic and metabolomic profile by TMT-labeled quantitative proteomics and untargeted metabolomics between the tramadol and the control group. Proteomic analysis revealed 31 differential expressed serum proteins ( 9 increased and 22 decreased) in tramadol-treated mice (oral, $50 \mathrm{mg} / \mathrm{kg}, 5$ weeks) as compared with the control ones. Bioinformatics analysis showed that the dysregulated proteins mainly included: enzyme inhibitor-associated proteins (i.e. apolipoprotein C-III (Apoc-III), alpha-1-antitrypsin 1-2 (Serpina 1b), apolipoprotein C-II (Apoc-II), plasma protease $\mathrm{C} 1$ inhibitor, inter-alpha-trypsin inhibitor heavy chain $\mathrm{H} 3$ (itih3)); mitochondriarelated proteins (i.e. 14-3-3 protein zeta/delta (YWHAZ)); cytoskeleton proteins (i.e. tubulin alpha-4A chain (TUBA4A), vinculin (Vcl)). And we found that the differential expressed proteins mainly involved in the pathway of the protein digestion and absorption. Metabolomics analysis revealed that differential expressed metabolites mainly involved in protein ingestion and absorption, fatty acid biosynthesis, steroid hormone biosynthesis and bile secretion. Our overall findings revealed that chronic exposure to tramadol changed the proteomic and metabolomic profile of mice. Moreover, integrated proteomic and metabolomic revealed that the protein digestion and absorption is the common enrichment KEGG pathway. Thus, the combination of proteomics and metabolomics opens new avenues for the research of the molecular mechanisms of tramadol toxicity.

Tramadol is a central analgesic drug and a low-affinity opioid receptor agonist, which is often used for the treatment of moderate to severe pain ${ }^{1,2}$. Tramadol is metabolized into O-demethyltramadol and N-demethyltramadol, of which O-demethyltramadol has stronger pharmacological activity than the original drug ${ }^{3}$. Tramadol has analgesic and other functions by acting in two ways: O-demethylated metabolites with bio activity, and the synergistic effects of opioid and non-opioid mechanisms ${ }^{4}$.

Although tramadol has been an effective and well-tolerated agent for the management of moderately several acute or chronic pain ${ }^{3}$, its adverse effects have always been a concern for researchers. The untoward effects of tramadol include confusion, dizziness, seizures, drowsiness, and respiratory depression ${ }^{4,5}$. Especially after a long period of high doses of tramadol, more severe side effects include angioedema, the increased effect of anticoagulants, hypoglycemia ${ }^{6,7}$ and serotonin toxicity ${ }^{1}$. Some literature revealed that opioids abuse may result in structural changes and apoptosis of neurons $\mathrm{s}^{8,9}$. Zhou had pointed out that chronic exposure to tramadol could induce toxic effect to the neurotransmitters of zebrafish ${ }^{10}$. Mohamed reported that chronic exposure to tramadol induced oxidative damage, inflammation, and apoptosis on the cerebrum of rats ${ }^{4}$. To the best of our knowledge, some literature have revealed that chronic tramadol administration is related to the provoking production of reactive oxygen species (ROS) ${ }^{11-13}$.

\footnotetext{
${ }^{1}$ School of Forensic Medicine, China Medical University, No. 77, Puhe Road, Shenyang North New Area, Shenyang 110122, Liaoning Province, People's Republic of China. ${ }^{2}$ School of Fundamental Sciences, China Medical University, Shenyang 110014, People's Republic of China. ${ }^{3}$ Jacobs University, 28759 Bremen, Germany. ${ }^{4}$ These authors contributed equally: Shukun Jiang and Guojie Liu. ${ }^{\varpi}$ email: Ingao@cmu.edu.cn
} 
Metabolomics and proteomics, as components of systems biology, help to analyze the tramadol toxic mechanism from the perspective of organism integrity ${ }^{14,15}$. Moreover, the advent of advanced quantitative proteomics techniques allows the systematic study of changes in the expression profile of proteins that are static or perturbed $^{15,16}$.

Although brain tissues are the best samples for laboratory research on central nervous system disorders such as addiction, blood can be collected much more easily and at much lower risks ${ }^{17}$. Importantly, it contains nearly the entire proteome of the human body ${ }^{17}$. Moreover, the effects of long term exposure to tramadol on protein and metabolite in serum has not been known. To mimic the long exposure to tramadol, mice were exposed to $50 \mathrm{mg} / \mathrm{kg}$ level of tramadol for 35 days. Blood plasma was then used for tandem mass tags (TMT) quantitative proteomics and untargeted metabolomics. In the present study, we aim to observe the proteomic and metabolomic profile to provide the fundamental for the tramadol toxicity in serum.

\section{Materials and methods}

Chemical reagents. Tramadol was purchased from Shanghai Macklin Biotechnology Co., Ltd. (Shanghai, China). LC-MS/MS testing was conducted by Shanghai Bioprofile Technology Co. Ltd. Mouse Tubulin Alpha 4A (TUBa4A) ELISA kit, mouse Vinculin (Vcl) ELISA kit, mouse Inter-alpha-trypsin inhibitor heavy chain H3 (itih3) ELISA kit, Mouse Alpha-1-antitrypsin 1-2 (Serpina1b) ELISA kit, Mouse Transthyretin (Ttr) ELISA kit, Mouse Haptoglobin (Hp) ELISA kit, Mouse Retinol-binding protein 4 (RBP-4) ELISA kit, Mouse Apolipoprotein C-III (ApocIII) ELISA kit, Mouse Plasma protease C1 inhibitor (Serping) ELISA kit, Mouse Carboxypeptidase B2 (CPB2) ELISA kit, Mouse 14-3-3 protein zeta/delta (YWHAZ) ELISA kit, and Mouse Hemopexin (Hpx) ELISA kits were purchased from Shanghai Enzyme-linked Biotechnology Co., Ltd. (Shanghai, China).

Animal treatment and sample collection. $16 \mathrm{~km}$ male mice (6 weeks old, $35 \pm 5 \mathrm{~g}$, Laboratory Animal Centre of China Medical University) were used in the studies. The mice were randomly assigned to a tramadol group ( $\mathrm{H}$ group, 8 mice) or control group ( $\mathrm{N}$ group, eight mice) and were housed in a controlled environment (20-22 ${ }^{\circ} \mathrm{C}$; 12 -h light:dark on a reversed light cycle) for 1 week before the studies. Mice had unlimited access to water and food in their home cages. Mice received physiological saline $(20 \mathrm{~mL} / \mathrm{kg})$ or tramadol $(2.5 \mathrm{mg} /$ $\mathrm{mL}$ ) via oral gavage daily ( $50 \mathrm{mg} / \mathrm{kg} /$ day) for 35 days in group. Mice were fasted for $24 \mathrm{~h}$ and anesthetized by intraperitoneal injection with pentobarbital prior to blood collection. Heart blood of each mouse was collected and centrifuged at $3000 \mathrm{rpm}$ for $15 \mathrm{~min}$ at $4{ }^{\circ} \mathrm{C}$ to obtain the serum, for proteomics and metabolomics analysis.

Serum proteomics analysis. Protein extraction. Approximately $100 \mu \mathrm{L}$ of serum was taken from each sample group (the tramadol group and the control group), which was then added into $600 \mu \mathrm{L} 8 \mathrm{M}$ urea. Subsequently, sonicated, and the supernatant was extracted after centrifugation at $20,000 \times g$ for $15 \mathrm{~min}$ at $4{ }^{\circ} \mathrm{C}$. Protein quantification was performed using the bicinchonic acid assay. About $15 \mu \mathrm{g}$ of protein was obtained from the two different groups for SDS-PAGE analysis to evaluate the quantitative accuracy and quantify protein extraction.

Protein digestion and peptide desalination. $300 \mu \mathrm{g}$ of sample from each group was taken for protein digestion. DTT was added into the protein sample, and the final concentration was $100 \mathrm{mM}$. Bathe in boiling water for $5 \mathrm{~min}$ and cool to room temperature. After adding $200 \mu \mathrm{L}$ of urea (UA) buffer (8 M UA and $150 \mathrm{mM}$ Tris- $\mathrm{HCl}$, $\mathrm{pH} 8.0$ ), the samples were loaded on a $10 \mathrm{kDa}$ ultra filtration centrifuge tube, followed by centrifugation at $12,000 \mathrm{~g}$ for $15 \mathrm{~min}$, and the filtrate was discarded (this step was repeated once). Subsequently, samples and $100 \mu \mathrm{L}$ of iodoacetamide alkylation (IAA) (50 mm IAA in UA) were shaken for 1 min at $600 \mathrm{RPM}$, then were placed in a centrifuge at $12,000 \mathrm{~g} \times 10 \mathrm{~min}$ after $30 \mathrm{~min}$ at room temperature in the dark. Next, another $100 \mu \mathrm{L}$ UA buffer was added, and the sample was centrifuged at $12,000 \mathrm{~g} \times 10 \mathrm{~min}$. We repeated the process twice. This was followed by a $100 \mu \mathrm{L} 100 \mathrm{~mm} \mathrm{NH}_{4} \mathrm{HCO}_{3}$ buffer (Sigma), which was centrifuged at 14,000 $\mathrm{g}$ for $10 \mathrm{~min}$. We

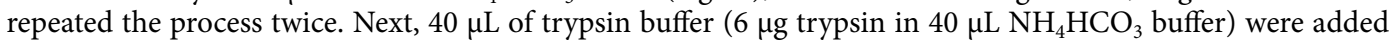
into the sample. The sample was shaken at $600 \mathrm{RPM}$ for $1 \mathrm{~min}$ and incubated at $37^{\circ} \mathrm{C}$ for $16-18 \mathrm{~h}$. The collection tube was replaced and then centrifuged for $10 \mathrm{~min}$ at $12,000 \mathrm{~g}$. The filtrate was collected and an amount of $0.1 \%$ trifluoroacetic acid (TFA) solution was added, followed by desalination in a C18 Cartridge (Sigma-Aldrich) and OD280 peptide quantification. Finally, approximately $150-180 \mu \mathrm{g}$ peptides were collected.

TMT peptide labeling, fractionation and LC-MS/MS analysis. This part of the content was operated according to the method reported by Zhang ${ }^{18}$, and the specific method parameters are shown in "Supplement file". The schematic for the TMT-labeling strategy used for the samples as shown in Supplementary Fig. 1.

Database search and protein quantification. Database search is similar with the method reported by Zhang ${ }^{18}$, the specific method parameters are shown in "Supplement file". False discovery rate (FDR) for peptide and protein identification was set to $1 \%$. The TMT reporter ion intensity was applied for quantification. The relative quantitative protein analysis of samples was performed using MaxQuant algorithms (http://www.maxquant.org, VERSION 1.6.0.16) ${ }^{19}$. The mass spectrometry proteomics data have been deposited to the ProteomeXchange Consortium (http://proteomecentral.proteomexchange.org) via the iProX partner repository with the dataset identifier PXD019233 20.

Bioinformatics analysis. Analyses of bioinformatics data were obtained by the Perseus software program ${ }^{21}$, Microsoft Excel, and R statistical computing software. Differential expressed proteins (DEPs) were screened in 
the tramadol group and the control group, with the cut-off of ratio fold change of $>1.20$ or $<0.83$ in expression and $\mathrm{P}$ values $<0.05$. In addition, DEPs in the tramadol group vs. the control groups were identified using student's T-Test. The proteins with significant difference (FDR q <0.01) were clustered by Hierarchical cluster analysis (Euclidean distance). Information was extracted from UniProtKB/Swiss-Prot, Kyoto Encyclopedia of Genes and genome (KEGG) and Gene Ontology (GO) for functional enrichment analysis of identified proteins ${ }^{22,23}$. Fisher's exact test was used for GO and KEGG enrichment analysis, and Benjamini-Hochberg false discovery rate (BH-FDR) correction for multiple tests was obtained. The term GO could be divided into three categories: biological process (BP), cellular component (CC), and molecular function (MF) ${ }^{24}$. The construction of PPI networks was also performed by the STRING database with the Cytoscape software program ${ }^{25}$.

Untargeted metabolomics analysis. Metabolite extraction. $100 \mu \mathrm{L}$ of water and $800 \mu \mathrm{L}$ of methanol/ acetonitrile $(1: 1, \mathrm{v} / \mathrm{v})$ were added for each sample $(100 \mu \mathrm{L})$. Ultrasound was performed at low temperature for $30 \mathrm{~min}$, twice, incubated at $-20{ }^{\circ} \mathrm{C}$ for $1 \mathrm{~h}$, and centrifuged at $4{ }^{\circ} \mathrm{C}$ at $13,000 \mathrm{RPM}$ for $15 \mathrm{~min}$, the supernatant was taken for freeze-drying and stored at $-80{ }^{\circ} \mathrm{C}$ for later use. During mass spectrometry, $100 \mu \mathrm{L}$ acetonitrile solution was added (acetonitrile: water $=1: 1, \mathrm{v} / \mathrm{v}$ ) was redissolved, vortex oscillated, centrifuged at $14,000 \mathrm{~g}$ at $4{ }^{\circ} \mathrm{C}$ for $15 \mathrm{~min}$, and the supernatant was injected into the LC-MS/MS system analysis.

Sample analysis and data preprocessing. This part was shown in "Supplement file". The data preprocessing was performed according to the method reported by $\mathrm{Gao}^{26}$. The resulting matrix was imported into SIMCA-P (version 13.0, Umetrics, Sweden) for unsupervised principal component analysis (PCA) and orthogonal partial least square discriminant analysis (OPLS-DA)after mean centering and unit variance scaling. We applied uni-variate analysis ( $\mathrm{t}$-test) to calculate statistical significance (P-value). Metabolites with variable importance in the project $(\mathrm{VIP})>1$, P-value $<0.05$ were considered differential metabolites. Metabolites of interest were filtered based on values of VIP, $|\log 2 \mathrm{FC}|>1(\mathrm{FC}>2$ or $\mathrm{FC}<0.5$ and $\mathrm{P}$-value $<0.05)$. Volcano plots were utilized to illustrate the distribution of differential metabolites.For clustering heat maps, data were normalized using z-scores of the intensity areas of differential metabolites and were plotted using the R package 27 "pheatmap".

Data quality evaluation in untargeted metabolomics analysis. Quality control samples (QCs) were obtained by pooling equal aliquots of each serum sample. QC samples are used to balance the chromatography-mass spectrometry system, to determine the state of the instrument, and to evaluate the stability of the system throughout the experiment. After mixing thoroughly, the QCs were analyzed consistently with real samples. Before the batch analysis, five QCs were first tested to stabilize the analytical system, and the acquired data were removed before data processing. All QCs were inserted randomly through the analytical batch to monitor the robustness of sample preparation and the stability of instrument analysis.

Elisa. Carboxypeptidase B2 (CPB2), inter-alpha-trypsin inhibitor heavy chain H3 (Itih3), Alpha-1-antitrypsin1-2 (Serpina1b), plasma protease C1 inhibitor (serping), Tubulin alpha-4A chain (Tuba4a), Vinculin (Vcl) and apolipoprotein c-III (ApocIII), haptoglobin (Hp), Transthyretin (Ttr), Retinol-binding protein 4(RBP4), 14-3-3 protein zeta/delta (YWHAZ), and Hemopexin (Hpx) Elisa kits were used to analyze the serum of ApocIII, Ttr, RBP-4,TUBa4A, Serpina1b, Vcl, Hp,YWHAZ, Hpx, CPB2, Itih3 and serping level following the manufacture's instruction.

Statistical analysis. The levels of ApocIII, Ttr, RBP-4, TUBa4A, Serpina1b, Vcl, Hp, YWHAZ, Hpx, CPB2, Itih3 and serping were present as mean \pm standard deviation (SD) and analyzed by SPSS 26.0 (SPSS Inc., Chicago, USA). The student's T-test was used and $\mathrm{P}<0.05$ was considered statistically significant.

Ethics approval. All experimental procedures were conducted in accordance with the guidelines of the animal care institution and approved by the laboratory animal management committee of China Medical University (2019288).

\section{Results}

Proteomics results. Information on proteome data was derived as follows: number of peptide-spectral matches (i.e., several spectra hits for the same peptide), unique peptide number, protein groups, and quantified proteins, which were 13,776, 2557, 469, and 443, respectively. All quantified proteins were shown in Table 1S. A boxplot of normalized density is shown in Fig. 1A. In addition, pairwise Pearson's correlation coefficients from all six samples ( 3 replicates $\times 2$ groups) were used to assess the relative quantitative reproducibility of the proteins and the results showed high reproducibility $(\mathrm{R}>0.94)$ (Fig. 1B).

Total of 31 differential expressed proteins (DEPs) were found, of which nine proteins were up-regulated and 22 proteins were down-regulated, as shown in Fig. 1C,D and Table 1.

All DEPs were classified into different Gene Ontology (GO) terms, biological process (BP), cell component (CC) and molecular function (MF) (Fig. 2A).

The top GO terms for BP enriched by DEPs in $\mathrm{H}$ group vs. $\mathrm{N}$ group were the regulation of proteolysis. The prominent GO CC categories that were enriched by these proteins included the extracellular region. The DEPs were associated with structural constituents of the cytoskeleton, actin filament binding, enzyme regulator activity, and structural molecule activity. In the PPI network of the DEPs, proteins such as Cpb2 (down-regulated), HpX (down-regulated), Itih3 (down-regulated), Serpinalb (up-regulated), serping1(down-regulated), Apom (up-regulated), Tuba4a (down-regulated), $\mathrm{Vcl}$ (down-regulated) and ApocIII (up-regulated) were mainly take 
A
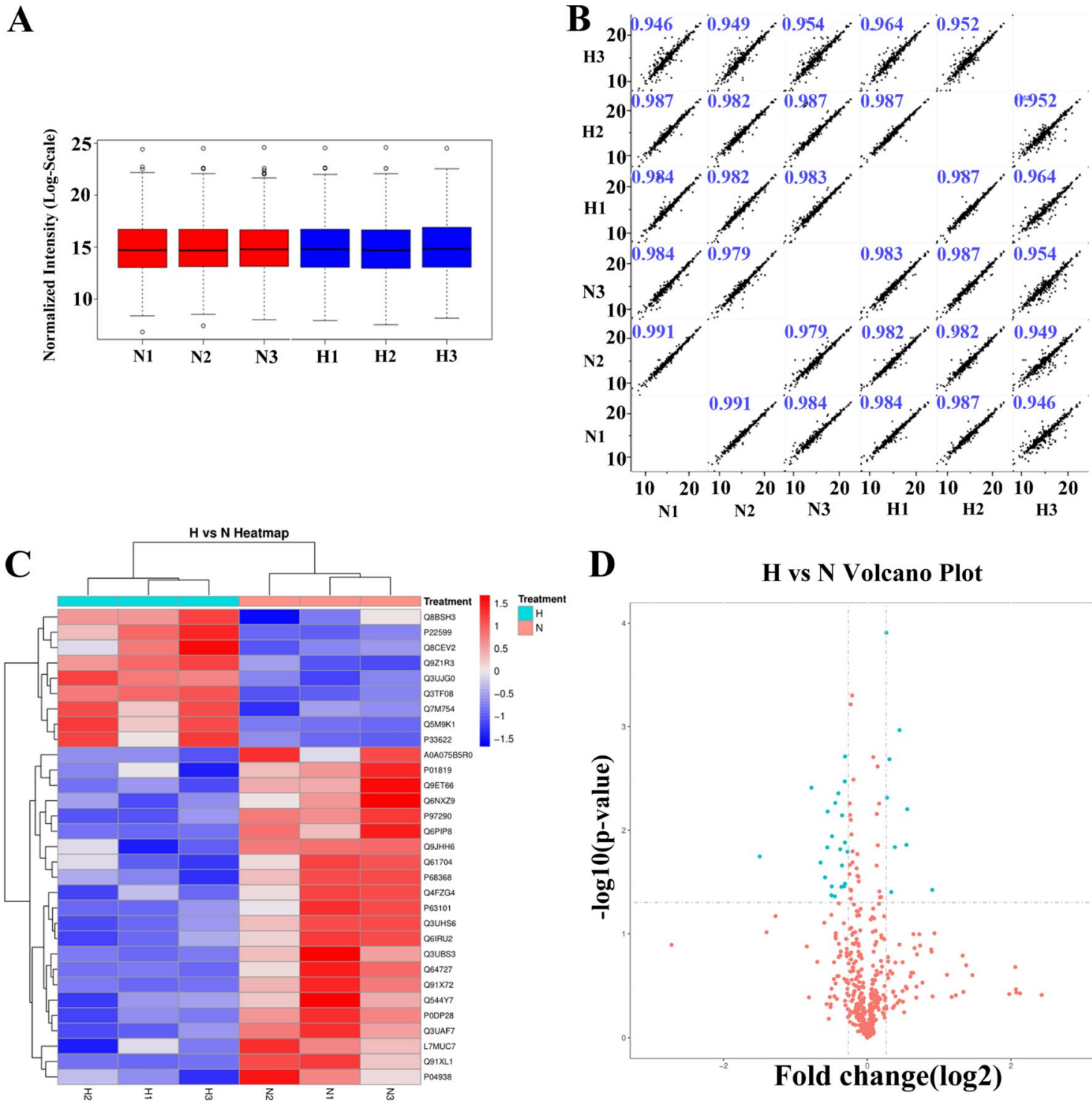

Figure 1. Volcano plots and heatmaps of proteins with differential expression between the tramadol group and the control group. (A) Boxplot of normalized density. (B) Pearson's correlation of normalized densities; (C) Cluster analyses of the control group and the tramadol group (blue represents dysregulated, gray means that there is no distinguished difference between the tramadol group and the control group); (D) Volcano plots and heatmaps of proteins with differential expression $(\mathrm{FC}>1.2$ or $\mathrm{FC}<0.833$, $\mathrm{P}$ value $<0.05)$.

part in the following KEGG pathways: protein digestion and absorption, the peroxisome proliferator-activated receptor (PPAR) signaling pathway, cardiac muscle contraction and adherens junctions (Fig. 2).

Some feature of DEPs' changes were similar to other toxicological studies of the substance subject to abuse (Table 2) $)^{10,17,28-33}$.

While the expression level of ApocIII, Ttr, RBP-4 and TUBa4A was up-regulated, the expression level of Serpinalb, Vcl, Hp, YWHAZ, Hpx, CPB2, Itih3 and serping were down-regulated by Elisa (Fig. 3).

Metabolomics results. The results of QC control data showed that the response strength and retention time of each chromatographic peak overlapped basically. The Pearson's correlation coefficient among QC samples was calculated based on the peak area value and showed that the variation caused by instrument error is small and the data quality is reliable (Supplementary Fig. 3).

There are 29 differential expressed metabolites (DEMs) between the $\mathrm{H}$ and $\mathrm{N}$ group (Fig. 4 and Table 3).

The PCA results of all groups did not show a satisfactory separation of data between the tramadol exposure group and the control group, that was shown in Supplementary Fig. 4 . The variance $\left(R^{2}\right)$ and model predictability $\left(\mathrm{Q}^{2}\right)$ for the OPLS-DA were calculated to be 0.992 and 0.699 under negative mode, and $\mathrm{R}^{2}$ and $\mathrm{Q}^{2}$ for the OPLSDA were calculated to be 0.947 and 0.529 under positive mode. In conclusion, the model had good reliability and predictability as shown in Fig. 5. 


\begin{tabular}{|c|c|c|c|c|c|}
\hline Identified protein names & Protein IDs & Score & FC & $\mathbf{P}$ & Molecular function \\
\hline Glyceraldehyde-3-phosphate dehydrogenase & Q8CEV2 & 13.161 & 1.873294763 & 0.03772571 & Oxidative reductase activity \\
\hline Apolipoprotein C-III & A0A0R4J1N3 & 323.31 & 1.305433615 & 0.01459075 & Enzyme inhibitor activity \\
\hline Retinol-binding protein 4 & H7BWY6 & 323.31 & 1.202682984 & 0.00012348 & $\begin{array}{l}\text { Retinol transporter activity; lipid transporter activity; lipid } \\
\text { binding; retinol binding }\end{array}$ \\
\hline Transthyretin & Q5M9K1 & 323.31 & 1.470107619 & 0.00626931 & Thyroid hormone-binding protein \\
\hline Alpha-1-antitrypsin 1-2 & P22599 & 142 & 1.209979 & 0.00485745 & Enzyme inhibitor activity; peptidase inhibitor activity \\
\hline Tropomyosin alpha-1 chain & Q8BSH3 & 26.514 & 1.260379028 & 0.03965987 & Structural constituent of cytoskeleton \\
\hline Apolipoprotein C-II & Q3UJG0 & 7.7292 & 1.364705953 & 0.00107894 & $\begin{array}{l}\text { Enzyme activator activity; enzyme regulator activity; } \\
\text { molecular function regulator }\end{array}$ \\
\hline Apolipoprotein M & Q9Z1R3 & 94.515 & 1.237485713 & 0.00206508 & Antioxidant activity; anion binding \\
\hline Tubulin alpha-4A chain & A0A0A0MQA5 & 7.6679 & 0.711702291 & 0.01147102 & Structural constituent of cytoskeleton \\
\hline 14-3-3 protein zeta/delta & A0A2I3BQ03 & 33.122 & 0.637214709 & 0.02060388 & $\begin{array}{l}\text { Cadherin binding; enzyme binding; ubiquitin protein ligase } \\
\text { binding; cell adhesion molecule binding; protein kinase } \\
\text { binding }\end{array}$ \\
\hline Cofilin-1; Cofilin-2 & Q544Y7 & 24.855 & 0.801105533 & 0.03488061 & Actin filament binding; cytoskeletal protein binding \\
\hline Major urinary protein 6; major urinary proteins 11 and 8 & A2CEK7 & 20.781 & 0.710121107 & 0.03493991 & $\begin{array}{l}\text { Transporter activity; transmembrane receptor protein tyros- } \\
\text { ine kinase activity; kinase activity }\end{array}$ \\
\hline Actin, cytoplasmic 1 & Q3UAF7 & 217.53 & 0.805697691 & 0.00338196 & $\begin{array}{l}\text { Anion binding; carbohydrate derivative binding; purine } \\
\text { ribonucleoside triphosphate binding; ATP binding }\end{array}$ \\
\hline Filamin-A & B7FAV1 & 90.22 & 0.783548733 & 0.02197410 & $\begin{array}{l}\text { Actin filament binding; actin binding; cytoskeletal protein } \\
\text { binding }\end{array}$ \\
\hline Peptidase inhibitor 16 & E9QNE5 & 115.97 & 0.8264383 & 0.01615836 & Enzyme inhibitor activity; peptidase inhibitor activity \\
\hline Calmodulin-like protein 3 & P0DP28 & 217.53 & 0.583894819 & 0.00388330 & $\begin{array}{l}\text { Enzyme binding; phosphatidylinositol 3-kinase binding; } \\
\text { glutamate receptor binding; calcium-dependent protein } \\
\text { binding; G-protein coupled receptor binding }\end{array}$ \\
\hline Talin-1 & Q3UHS6 & 54.399 & 0.734627417 & 0.00545763 & $\begin{array}{l}\text { Actin filament binding; structural constituent of cytoskel- } \\
\text { eton; cytoskeletal protein binding }\end{array}$ \\
\hline Plasma protease $\mathrm{Cl}$ inhibitor & P97290 & 103.99 & 0.807485012 & 0.00194138 & Enzyme inhibitor activity; peptidase inhibitor activity \\
\hline Haptoglobin & Q3UBS3 & 110.08 & 0.35444712 & 0.01797707 & Hemoglobin binding; serine-type endopeptidase activity \\
\hline Inter-alpha-trypsin inhibitor heavy chain $\mathrm{H} 3$ & Q61704 & 183.26 & 0.806781264 & 0.03295815 & Enzyme inhibitor activity; peptidase inhibitor activity \\
\hline Vinculin & Q64727 & 323.31 & 0.769615002 & 0.01528594 & Actin filament binding; cytoskeletal protein binding \\
\hline Tropomyosin alpha- 4 chain & Q6IRU2 & 45.874 & 0.680481412 & 0.01468016 & Actin filament binding; cytoskeletal protein binding \\
\hline Hemopexin & Q91X72 & 323.31 & 0.756880662 & 0.00440384 & $\begin{array}{l}\text { Heme transporter activity; cofactor transporter activity; } \\
\text { organic cyclic compound binding }\end{array}$ \\
\hline Carboxypeptidase B2 & Q9JHH6 & 44.331 & 0.806708298 & 0.01316768 & $\begin{array}{l}\text { Zine ion binding; metallopeptidase activity; hydrolase } \\
\text { activity }\end{array}$ \\
\hline
\end{tabular}

Table 1. The differential expression proteins in $\mathrm{H}$ vs $\mathrm{N}$.

A

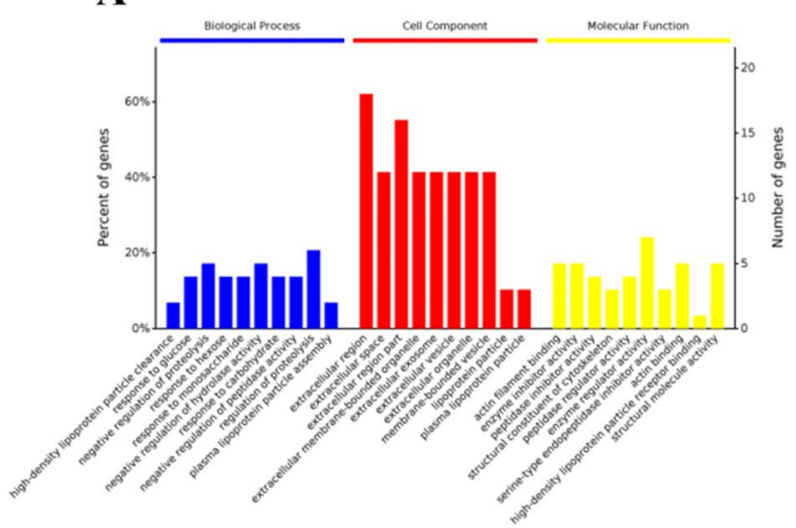

B

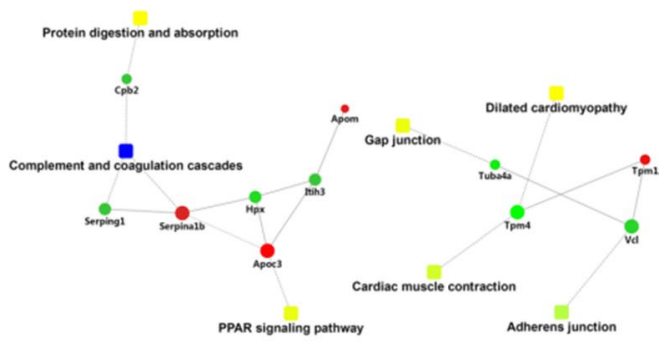

Figure 2. (A) Gene ontology (GO) term (including biological process, cellular component, and molecular function) enrichment for differential expressed proteins in the tramadol group and the control group. (B) Protein-protein interaction (PPI) networks of differential expressed proteins (DEPs) between the $\mathrm{H}$ and $\mathrm{N}$ group. Red nodes indicate up-regulated proteins and green nodes indicate down-regulated proteins. The rectangular node represents the KEGG pathway/biological process. The P-value is represented by a yellow-blue color gradient; the yellow color indicates a small P-value and the blue color indicates a large P-value. 


\begin{tabular}{|l|l|}
\hline Protein & Evidence from other drug researches \\
\hline Enzyme inhibitor-associated proteins & Alcohol $^{28}$ \\
\hline Apolipoprotein C-III (Apoc-III) & Alcohol $^{28}$ \\
\hline Apolipoprotein CII (Apoc-II) & Alcohol $^{29}$ \\
\hline Inter-alpha-trypsin inhibitor heavy chain H3 (itih3) & \multicolumn{2}{|l|}{} \\
\hline Mitochondria-related proteins & Tramadol $^{10}$ \\
\hline 14-3-3 protein zeta/delta (YWHAZ) & Nictoine \\
\hline Cytoskeleton proteins & Nicotine $^{32}$ \\
\hline Vinculin (Vcl) & \\
\hline Hemopexin (Hpx) & Amphetamine $^{33}$, heroin $^{17}$ \\
\hline Others & Alcohol $^{29}$, heroin $^{17}$ \\
\hline Haptoglobin (Hp) & Alcohol $^{29}$ \\
\hline Transthyretin (Ttr) &
\end{tabular}

Table 2. Summary of proteins modified by tramadol and one or more other substances of abuse.

The metabolomic profile of the $\mathrm{H}$ and $\mathrm{N}$ group is different (Fig. 6A). These DEMs were enriched for the KEGG pathway "biosynthesis of amino acids", "protein digestion and absorption", "valine, leucine and isoleucine biosynthesis" and "valine, leucine and isoleucine degradation" etc. (Fig. 6B).

Integrated analysis of metabolomics and proteomics. The DEPs and DEMs in the $\mathrm{H}$ and $\mathrm{N}$ group mainly were involved in nicotinate and nicotinamide metabolism, phenylalanine metabolism and the PPAR signaling pathway as shown in Fig. 7. From the Fig. 2S, these DEMs and DEPs were enriched for the KEGG pathway "biosynthesis of amino acids", "steroid hormone biosynthesis", "phenyalaine metabolism", "tyrosine metabolism", "nicotinate and nicotinamide metabolism", "focal adhesion". Moreover, the common enrichment KEGG pathway for DEPs and DEMs is "protein digestion and absorption" including the upregulation of L-isoleucine; L-valine; L-leucine; L-phenylalanine; L-glutamine and down-regulated Cpb2 by integrated analysis of metabolomics and proteomics.

\section{Discussion}

Although tramadol has become the most prescribed opioid worldwide, there are few assays validated the effect of chronic exposure to tramadol. To obtain the comprehensive system biology profile, two 'omics' pipelines utilizing LC-MS/MS instrumentation were developed and leveraged for this work. We found that 31 DEPs and 34 DEMs in the $\mathrm{H}$ and $\mathrm{N}$ groups. Moreover, KEGG pathway "protein digestion and absorption" was the key pathway in the effects of tramadol.

Given the serum is obtained easily in the clinic practice, and few reports focused on the effects of tramadol on proteins and metabolites in serum, so we chose the serum as the sample. In the present study, TMT-LC-MS/ MS-based quantitative proteome analysis showed protein changes in the metabolic process between the $\mathrm{H}$ and $\mathrm{N}$ groups. By bioinformatics analysis,we found that these proteins were classified into different Gene Ontology (GO) terms, biological process (BP), cell component (CC) and molecular function (MF) (Fig. 2A). The top GO terms for $\mathrm{BP}$ enriched by DEPs in $\mathrm{H}$ (the tramadol group) vs. $\mathrm{N}$ (the control) group were the regulation of proteolysis. The prominent GO CC categories that were enriched by these proteins included the extra-cellular region. Bioinformatics analyses also indicated that these DEPs were associated with structural constituents of the cytoskeleton, actin filament binding, enzyme regulator activity, and structural molecule activity.

To further investigate the functions of these DEPs, protein-protein interaction (PPI) networks were constructed for proteins with differential expression in the $\mathrm{H}$ and $\mathrm{N}$ group. As shown in Fig. $2 \mathrm{~B}$, these DEPs mainly take part in the following KEGG pathways: protein digestion and absorption, the peroxisome proliferatoractivated receptor (PPAR) signaling pathway, cardiac muscle contraction and adherens junctions. The DEPs in the PPI networks had a relatively high degree of connectivity, what's more, we validated these differential proteins by Elisa kit. The effect of long term exposure to tramadol was associate with protein digestion and absorption, the PPAR signaling pathway, cardiac muscle contraction and adherens junctions.

By comparing our experimental results with similar toxicological studies of the substance subject to abuse, we found similar DEPs shown in Table 3. Some DEPs (Apoc III, Apoc II, itih3, YWHAZ, Vcl, Hpx, Hp, Ttr, RBP-4) in our study were compared with those differential proteins induced by other addiction drug i.g. nicotine, alcohol, morphine, heroin, amphetamine, and tramadol in Table 3. Actin and Vcl were also down-regulated after nicotine abuse. The down-regulated $\mathrm{Hp}$ and up-regulated Ttr were also reported after alcohol and heroin abuse, what's more, variation trend of $\mathrm{Hp}$ and $\mathrm{Ttr}$ induced by tramadol was coordinate with the heroin addicts. Hp is a highly abundant plasma glycoprotein, its main function is to combine with free haemoglobin $(\mathrm{Hb})$ to form stable $\mathrm{Hp}-\mathrm{Hb}$ complexes. The bioinformatics analysis of $\mathrm{Hp}$ showed that $\mathrm{Hp}$ is important in the molecular regulation of inflammation, which also acts as an antioxidant, has antibacterial activity and plays a role in modulating many aspects of the acute phase response $\mathrm{s}^{3-36}$. The up-regulated RBP-4 was also found in the heroin addicts, suggested it may be a potential bio-marker for opioid abuse. The down-regulated Hpx was observed in our study, and Cecconi D reported similar results induced by nicotine abuse ${ }^{30}$. Hpx is a plasma protein belonging to positive acute-phase 
TUBa4A

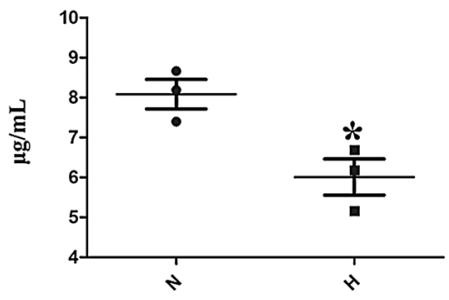

RBP-4

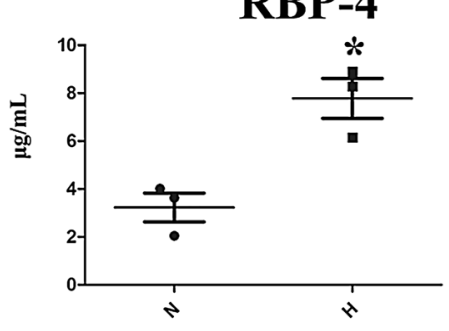

Hp

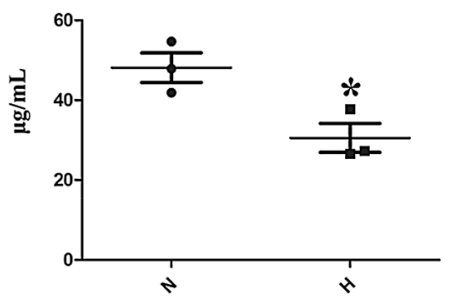

CPB2

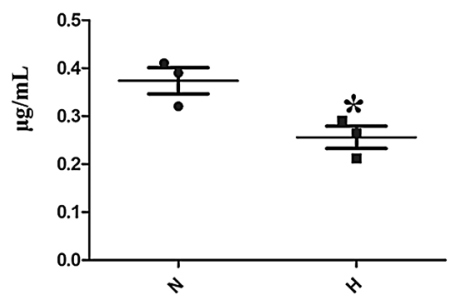

ApocIII

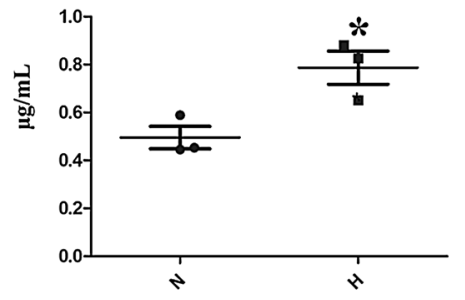

Serpina1b

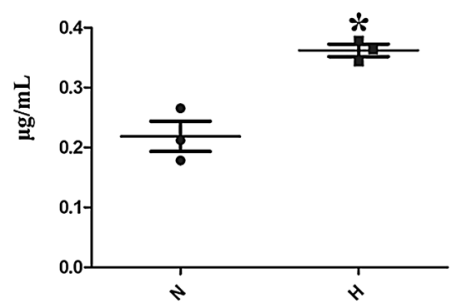

YWHAZ

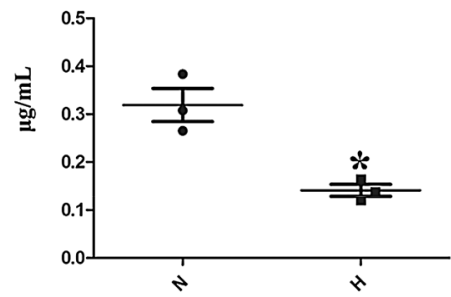

itih3

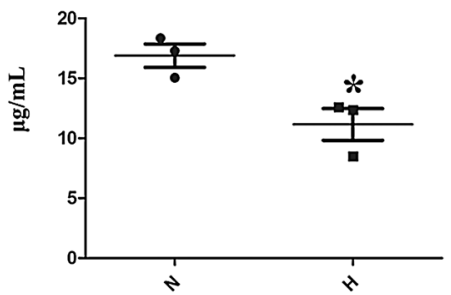

Ttr

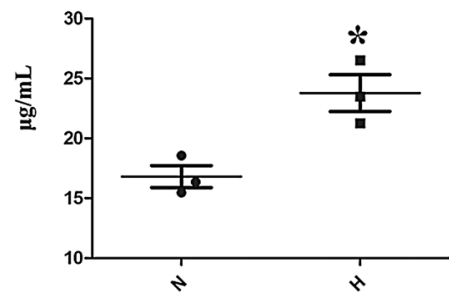

Vcl

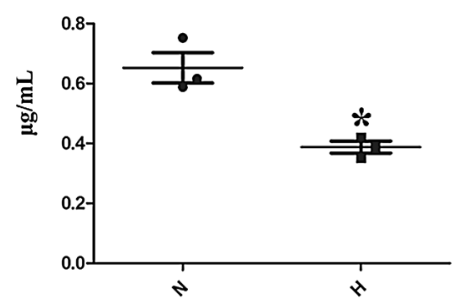

Hрx

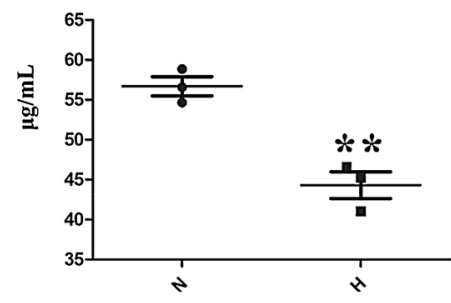

serping

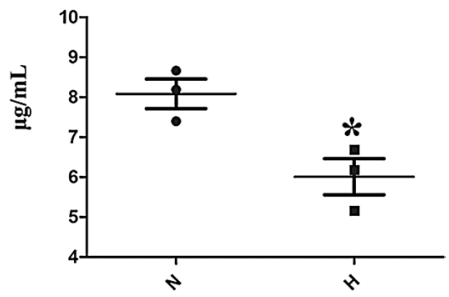

Figure 3. Elisa analyses of 12 differential expressed proteins. Changes in protein abundance shown by Elisa analysis and quantification of the proteins were highly consistent with the proteomic data of mice in serum. ${ }^{*} \mathrm{P}<0.05,{ }^{* * \mathrm{P}}<0.01$.

proteins that binds and transports haeme, thus preventing oxidative damage ${ }^{32}$. All the reports mentioned above, integrated with our findings, suggesting tramadol and these drugs caused similar changes in cellular activities and biological processes in the serum.

In order to obtain comprehensive profile change after exposure to tramadol, the changes in the metabolome were also be investigated. The metabolomic pattern was distinguished differences between the $\mathrm{H}$ and $\mathrm{N}$ groups. Creatine was suggested be correlated with kidney injury ${ }^{37}$. 1-Methylnicotinamide and nicotinamide are involved in nicotinate and nicotinamide metabolism. Moreover, they were suggested to be correlated with inflammation and oxidative damage ${ }^{3,26}$. Tryptophan can be transformed into 5-HT, which can also be transformed into indole acrylic acid and kynurenic acid ${ }^{38}$. We speculated the balance between tryptophan and 5-HT is disrupted by the up-regulation of indole acrylic acid and kynurenic acid. The branched-chain amino acids (BCAC, including L-isoleucine, L-leucine and L-valine) can stimulate the proliferation of monocytes, to enhance immune response. Rebholz suggested that an imbalance of BCAC may lead to immune damage, that is correlated with inflammation to some extent ${ }^{39}$. In addition, hydroxybutyric acid, glutamine and phenylalanine were suggested to be a correlation with neurotransmitter disruption ${ }^{6}$. Taken together, the enrichment KEGG pathway showed that these different metabolites take part in the biosynthesis of amino acids, protein digestion and absorption, valine, leucine and isoleucine biosynthesis and valine, leucine and isoleucine degradation. 
A

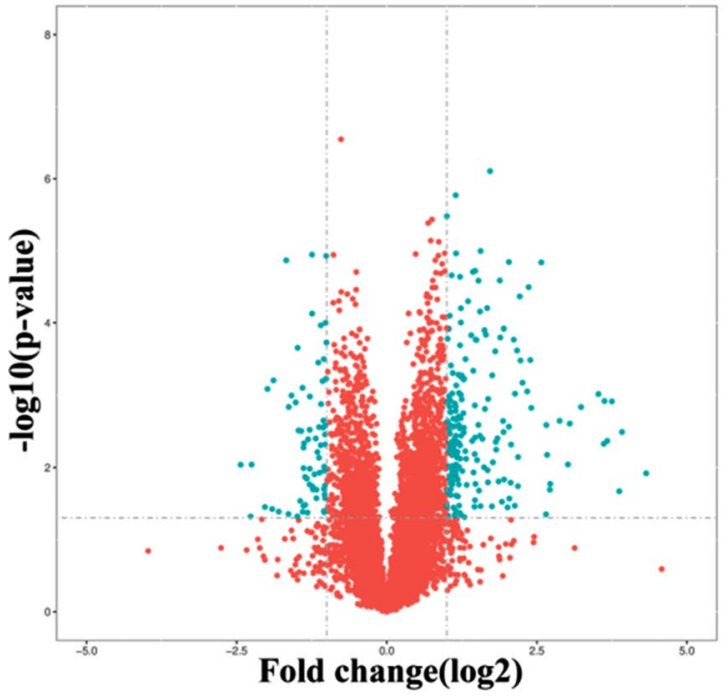

B

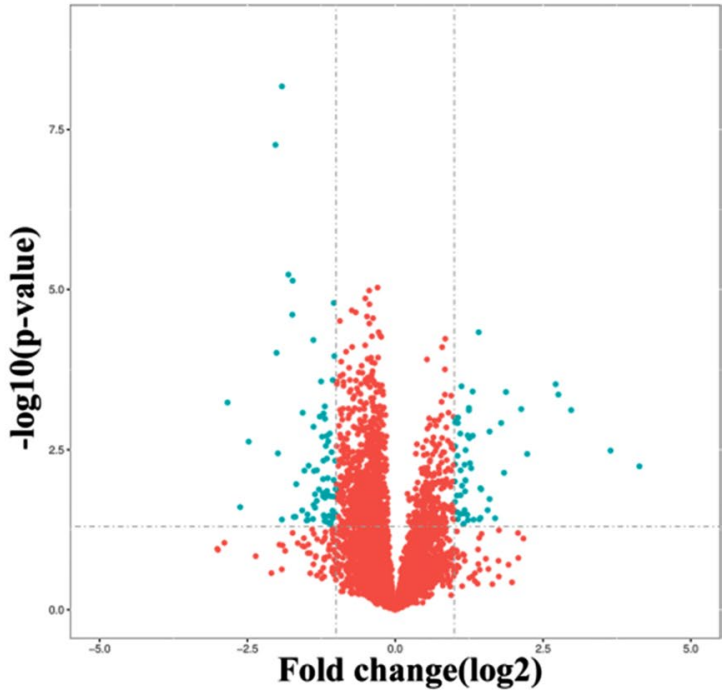

Figure 4. Volcano plots of metabolites with differential expression between the tramadol group and the control group (the (A) was obtained under negative ion mode; the (B) was obtained under positive ion mode, blue represents the distinguished metabolites $(\mathrm{FC}>2$ or $\mathrm{FC}<0.5$ and $\mathrm{P}$ value $<0.05$, $\mathrm{P}$ value is calculated by t-test formula).

\begin{tabular}{|c|c|c|c|c|c|c|}
\hline No. & Metabolite & VIP & $\mathrm{FC}(\mathrm{H} / \mathrm{N})$ & P value & Trend & Metabolic pathway \\
\hline 1 & 1-Methylnicotinamide & 1.43537 & 2.661641219 & $4.62 \mathrm{E}-05$ & $\uparrow$ & Nicotinate and nicotinamide metabolism \\
\hline 2 & L-Valine & 2.07855 & 1.599308079 & 0.000135188 & $\uparrow$ & Protein digestion and absorption; Valine, leucine and isoleucine biosynthesis \\
\hline 3 & L-Leucine & 1.98634 & 1.721599218 & 0.00056504 & $\uparrow$ & Protein digestion and absorption; Valine, leucine and isoleucine biosynthesis \\
\hline 4 & Acacetin & 1.31842 & 4.382382741 & 0.000732112 & $\uparrow$ & Flavone and flavonol biosynthesis \\
\hline 5 & Isoleucine & 4.60723 & 2.37196399 & 0.000764949 & $\uparrow$ & Protein digestion and absorption; Valine, leucine and isoleucine biosynthesis \\
\hline 6 & L-Carnosine & 1.40957 & 0.439065869 & 0.001036839 & $\downarrow$ & Histidine metabolism \\
\hline 7 & Cholesterol sulfate & 2.09954 & 0.67083571 & 0.001286897 & $\downarrow$ & Steroid hormone biosynthesis \\
\hline 8 & Corticosterone & 4.11877 & 0.517566612 & 0.001338819 & $\downarrow$ & Steroid hormone biosynthesis \\
\hline 9 & N-Cinnamoylglycine & 4.15159 & 3.106462903 & 0.001544527 & $\uparrow$ & The metabolites of glycine \\
\hline 10 & Nicotinamide & 3.96743 & 2.338651712 & 0.00188947 & $\uparrow$ & Nicotinate and nicotinamide metabolism; Vitamin digestion and absorption \\
\hline 11 & Benzoic acid & 1.80976 & 1.385671507 & 0.002237064 & $\uparrow$ & Degradation of aromatic compounds \\
\hline 12 & 20-Hydroxyeicosatetraenoic acid & 7.79954 & 0.715070263 & 0.002618757 & $\downarrow$ & Vascular smooth muscle contraction \\
\hline 13 & Kynurenic acid & 2.21865 & 2.074864706 & 0.003409024 & $\uparrow$ & The catabolites of tryptophan \\
\hline 14 & Phenylacetylglycine & 4.07605 & 1.973829943 & 0.004025549 & $\uparrow$ & Phenylalanine metabolism \\
\hline 15 & Mesaconic acid & 2.02296 & 0.763354166 & 0.0055795 & $\downarrow$ & C5-Branched dibasic acid metabolism \\
\hline 16 & DL-Beta-hydroxybutyric acid & 6.80608 & 0.621292252 & 0.006004963 & $\downarrow$ & Synthesis and degradation of ketone bodies \\
\hline 17 & L-Norleucine & 1.7419 & 2.275386538 & 0.009433052 & $\uparrow$ & Protein digestion and absorption; Valine, leucine and isoleucine biosynthesis \\
\hline 18 & Creatine & 1.08606 & 1.333084778 & 0.009437955 & $\uparrow$ & Arginine and proline metabolism; Glycine, serine and threonine metabolism \\
\hline 19 & L-Phenylalanine & 1.77148 & 1.434736131 & 0.010075828 & $\uparrow$ & Protein digestion and absorption; Phenylalanine metabolism \\
\hline 20 & Taurochenodeoxycholate & 2.53522 & 0.434162682 & 0.012439044 & $\downarrow$ & Bile secretion; Secondary bile acid biosynthesis; Primary bile acid biosynthesis \\
\hline 21 & Alpha-chaconine & 1.92791 & 0.771722033 & 0.015423328 & $\downarrow$ & Glycerophospholipid metabolism \\
\hline 22 & Pantothenate & 1.3127 & 1.487048436 & 0.015648029 & $\uparrow$ & Vitamin digestion and absorption; Pantothenate and CoA biosynthesis \\
\hline 23 & Eicosapentaenoic acid & 3.8208 & 1.271415181 & 0.019745226 & $\uparrow$ & Fatty acid biosynthesis \\
\hline 24 & L-Pipecolic acid & 1.40422 & 0.6239531 & 0.022905318 & $\downarrow$ & Lysine degradation \\
\hline 25 & Glutamine & 1.93396 & 1.300065997 & 0.023490107 & $\uparrow$ & $\begin{array}{l}\text { GABAergic synapse; D-glutamine and D-glutamate metabolism; Glutamatergic } \\
\text { synapse }\end{array}$ \\
\hline 26 & Cytidine & 1.49422 & 1.168843272 & 0.023943668 & $\uparrow$ & Pyrimidine metabolism \\
\hline 27 & 2-Hydroxy-4-methylpentanoate & 5.51063 & 1.960514129 & 0.025465597 & $\uparrow$ & Organic acid \\
\hline 28 & Indoleacrylic acid & 1.10864 & 1.885752926 & 0.03537406 & $\uparrow$ & The metabolites of tryptophan \\
\hline 29 & Dodecanoic acid & 2.28455 & 0.628753946 & 0.049332248 & $\downarrow$ & Fatty acid biosynthesis \\
\hline
\end{tabular}

Table 3. The distinguished different metabolites in $\mathrm{H}$ vs $\mathrm{N}$ groups. 
A
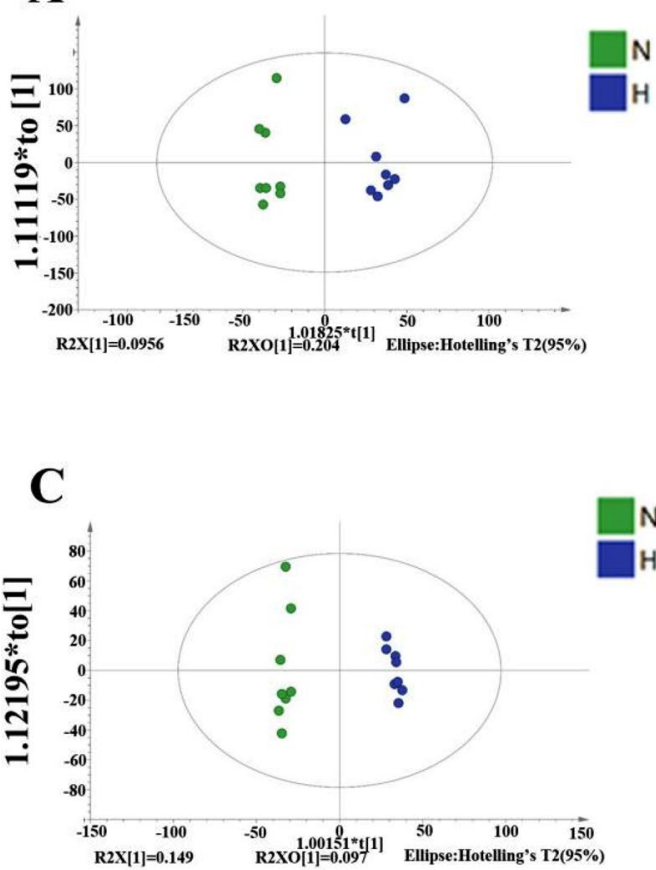

B Intercepts: $R 2=(0.0,0.89), Q 2=(0.0,-0.247)$

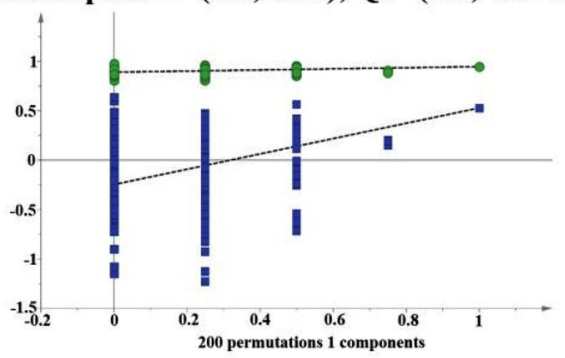

D Intercepts: $R 2=(0.0,0.965), Q 2=(0.0,-0.0986)$

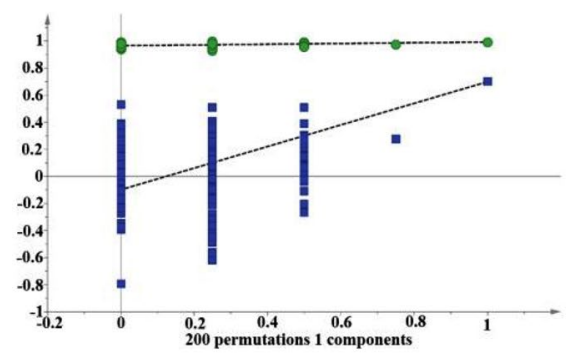

Figure 5. OPLS-DA Score Results and OPLS-DA valid figure of Mouse Serum Samples (A, C) represent the OPLS-DA score results; (A) was obtained under negative ion mode; $(\mathbf{C})$ was obtained under positive ion mode; $(\mathbf{C}, \mathbf{D})$ represent the OPLS-DA valid, (B) was obtained under negative ion mode, (D) was obtained under positive ion mode; intercepts: $\mathrm{R}^{2}$ and $\mathrm{Q}^{2}$ represent $\mathrm{y}$-intercept of $\mathrm{R}^{2}$ and $\mathrm{Q}^{2}$ regression lines. $\mathrm{N}$ represents the control group; $\mathrm{H}$ represents the tramadol group.

A

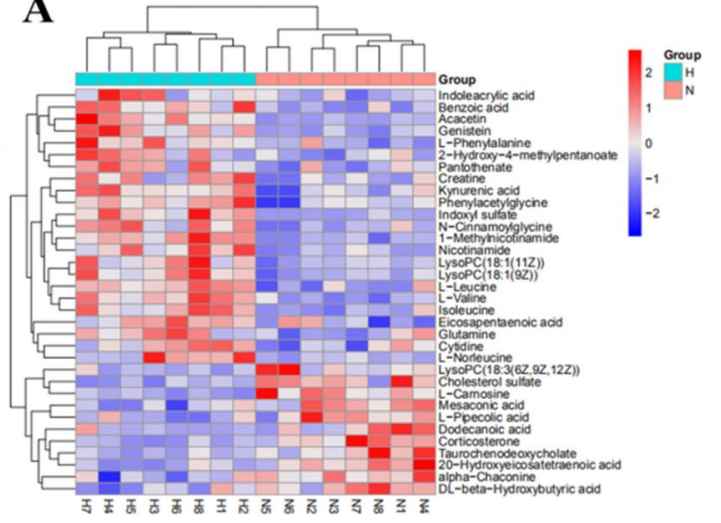

B

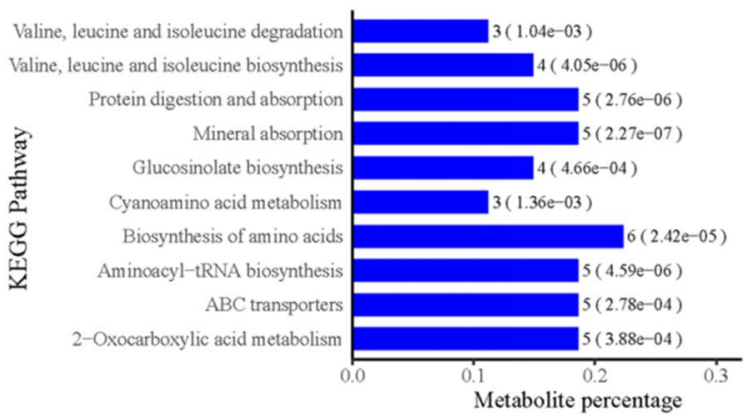

Figure 6. (A) Cluster analysis of the tramadol group and the control group (red represents up-regulated, blue represents down-regulated). (B) Enrichment KEGG pathway (top 10 between the tramadol group and the control group, $\mathrm{H}$ represents the tramadol group; $\mathrm{N}$ represents the control group).

In this context, the multi-omics was used to evaluate the effect of exposure to tramadol. In Fig. 7, we can see that the Nicotinate and nicotinamide metabolism and phenylalanine, tyrosine and tryptophan metabolism were affected after exposure to tramadol, that is coordinate with the relevance report ${ }^{2,4}$. Combination analyses of proteomics and metabolomics showed that $\mathrm{Cpb} 2$ could be used to elucidate the effect of tramadol. Cpb2 is a basic carboxypeptidase which can attenuate fibrinolysis and plays a role in regulating complement activation in vivo ${ }^{40,41}$, also shows anti-inflammatory activity in the presence of thrombin in vitro ${ }^{42}$. Carboxypeptidase $\mathrm{B}$ $(\mathrm{CpB})$ which cleaves carboxy-terminal lysine residues, abolished reactive oxygen species induced by oxidative stress $^{43}$. The downregulation of $\mathrm{Cpb} 2$ was speculated to weaken the defense of organs during oxidative damage caused by long-term exposure to tramadol.

Limitation are exist in the study, First, the serum proteome coverage reported is sub-optimum and we just suggested that a few differential protein can be used as potential key proteins to elucidate the toxicity of chronic exposure to tramadol, further investigations need to be performed for the identification of their modification in proteins. Second, for the ethical purpose, only eight mice, which can meet the purpose of metabolomics 


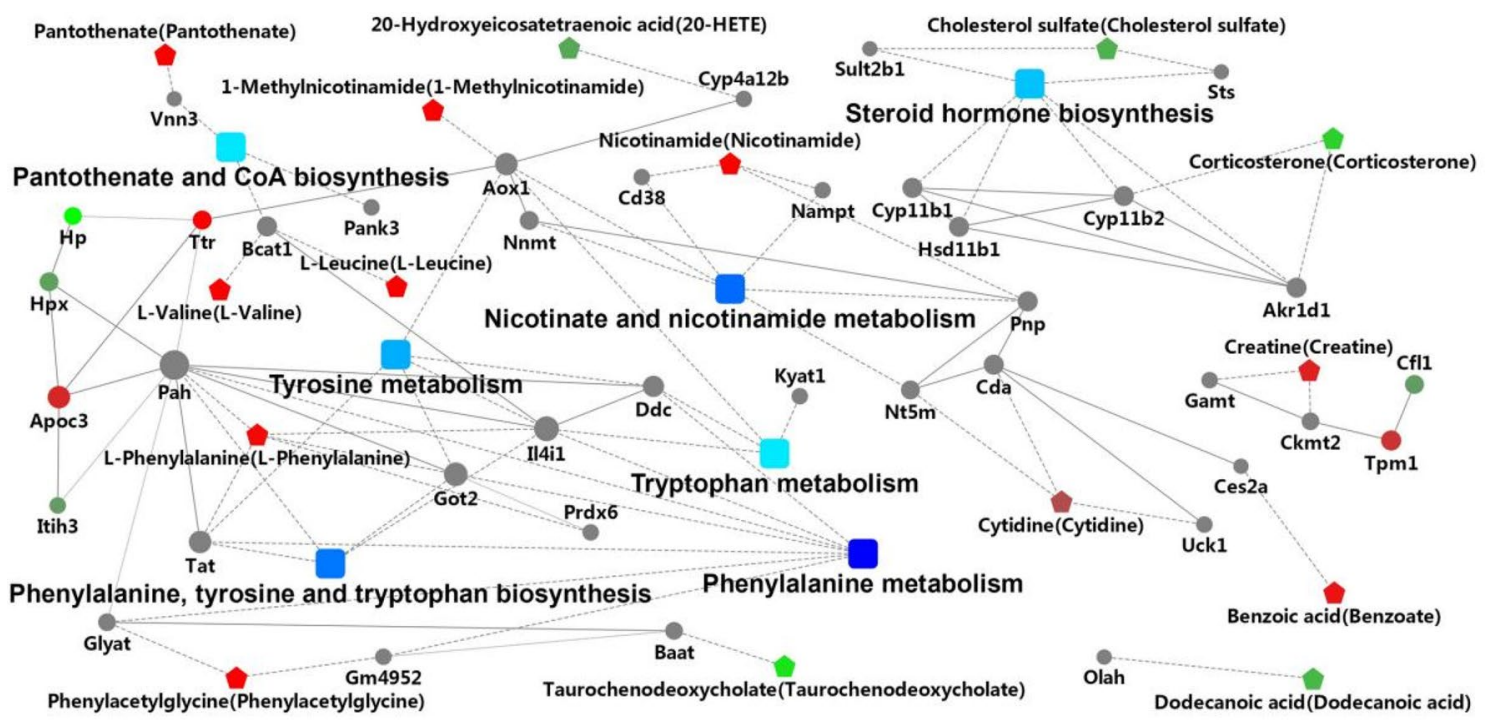

The KEGG pathway/biological process

The proteins in the tramadol group and the control group

$\square$ The metabolites in the tramadol group and the control group

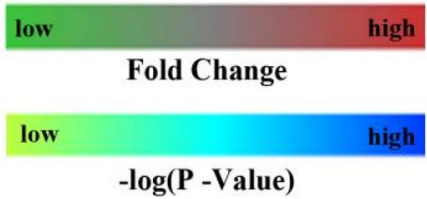

Figure 7. Combination analyses with proteomics and metabolomics. Red represents up-regulated and green represents down-regulated. The rectangular node represents the KEGG pathway/biological process. The circular and pentagon nodes represent the proteins and the metabolites in the tramadol group and the control group, respectively. The P-value is represented by a blue color gradient; the deeper blue color indicates a larger P-value.

analysis, were included in each group in the present study. In addition, quantitative studies of proteomics need to be performed to validate others DEPs not included in Fig. 3 after tramadol exposure in future research. As the high abundance proteins in serum were not removed, the low abundance proteins may not be detected, that result in the proteomics profile not the most comprehensive.

\section{Conclusion}

In conclusion, the proteomic and metabolomic profiles were significantly changed over a 5 weeks following exposure to tramadol, several protein and metabolite markers have been found to be significantly changed. These DEPs can be linked to protein digestion and absorption, the PPAR signaling pathway, cardiac muscle contraction and adherens junctions. These DEMs were enriched for the KEGG pathway "biosynthesis of amino acids", "protein digestion and absorption". Integrated analyses of proteomics and metabolomics, the common KEGG pathway is protein digestion and absorption. Collectively, our findings may provide the fundamental data for toxicity of tramadol in serum.

\section{Data availability}

The mass spectrometry proteomics data have been deposited to the ProteomeXchange Consortium (http://prote omecentral.proteomexchange.org) via the iProX partner repository with the dataset identifier PXD019233. All data are fully available without restriction.

Received: 10 August 2020; Accepted: 4 January 2021

Published online: 14 January 2021

\section{References}

1. Kimura, M., Obata, H. \& Saito, S. Antihypersensitivity effects of tramadol hydrochloride in a rat model of postoperative pain. Anesth. Analg. 115, 443-449 (2012).

2. Beakley, B. D., Kaye, A. M. \& Kaye, A. D. Tramadol, pharmacology, side effects, and serotonin syndrome: A review. Pain Phys. 18, 395-400 (2015)

3. Grond, S. \& Sablotzki, A. Clinical pharmacology of tramadol. Clin. Pharmacokinet. 43, 879-923 (2004).

4. Mohamed, H. M. \& Mahmoud, A. M. Chronic exposure to the opioid tramadol induces oxidative damage, inflammation and apoptosis, and alters cerebral monoamine neurotransmitters in rats. Biomed. Pharmacother. 110, 239-247 (2019).

5. Ryan, N. M. \& Isbister, G. K. Tramadol overdose causes seizures and respiratory depression but serotonin toxicity appears unlikely. Clin. Toxicol. (Phila.) 53, 545-550 (2015).

6. Shubhakaran, K. P. \& Khichar, R. J. Tramadol side effects: Seizure and hypoglycemia. Anesth. Essays Res. 10, 685-686 (2016).

7. Makunts, T., Ur, A., Atayee, S. \& Abagyan, R. Retrospective analysis reveals significant association of hypoglycemia with tramadol and methadone in contrast to other opioids. Sci. Rep. 9, 12490 (2019).

8. Liu, L. W. et al. Neuronal apoptosis in morphine addiction and its molecular mechanism. Int. J. Clin. Exp. Med. 6, 540-545 (2013). 
9. Mohamed, M., Ghaffar, H. M. \& El Husseiny, R. M. Effects of tramadol, clonazepam, and their combination on brain mitochondrial complexes. Toxicol. Ind. Health 31, 1325-1333 (2015).

10. Zhuo, H. Q., Huang, L., Huang, H. Q. \& Cai, Z. Effects of chronic tramadol exposure on the zebrafish brain: A proteomic study. J. Proteom. 75, 3351-3364 (2012).

11. Bameri, B. et al. Evidence for the involvement of the dopaminergic system in seizure and oxidative damage induced by tramadol. Int. J. Toxicol. 37, 164-170 (2018).

12. Abdel-Zaher, A. O., Abdel-Rahman, M. S. \& Elwasei, F. M. Protective effect of Nigella sativa oil against tramadol-induced tolerance and dependence in mice: Role of nitric oxide and oxidative stress. Neurotoxicology 32, 725-733 (2011).

13. Atici, S. et al. Liver and kidney toxicity in chronic use of opioids: An experimental long term treatment model. J. Biosci. 30, 245-252 (2005).

14. Xiao, S. \& Zhou, L. Gastric cancer: Metabolic and metabolomics perspectives (review). Int. J. Oncol. 51, 5-17 (2017).

15. Ronsein, G. E. et al. Parallel reaction monitoring (PRM) and selected reaction monitoring (SRM) exhibit comparable linearity, dynamic range and precision for targeted quantitative HDL proteomics. J. Proteom. 113, 388-399 (2015).

16. Zecha, J. et al. TMT labeling for the masses: A robust and cost-efficient, in-solution labeling approach. Mol. Cell Proteom. 18, 1468-1478 (2019).

17. Zhou, B. Y. et al. Serum proteomic analysis reveals high frequency of haptoglobin deficiency and elevated thyroxine level in heroin addicts. PLoS ONE 9, e95345 (2014).

18. Zhang, G. et al. Quantitative proteomics analysis reveals proteins and pathways associated with anthocyanin accumulation in, barley. Food Chem. 298, 124973 (2019).

19. Tyanova, S., Temu, T. \& Cox, J. The MaxQuant computational platform for mass spectrometry-based shotgun proteomics. Nat. Protoc. 11, 2301-2319 (2016).

20. Ma, J. et al. iProX: An integrated proteome resource. Nucleic Acids Res. 47, D1211-D1217 (2019).

21. Tyanova, S. et al. The Perseus computational platform for comprehensive analysis of (prote)omics data. Nat. Methods 13, 731-740 (2016).

22. Boutet, E. et al. UniProtKB/Swiss-Prot, the manually annotated section of the UniProt KnowledgeBase: How to use the entry view. Methods Mol. Biol. 1374, 23-54 (2016).

23. Kanehisa, M., Goto, S., Sato, Y., Furumichi, M. \& Tanabe, M. KEGG for integration and interpretation of large-scale molecular data sets. Nucleic Acids Res. 40, D109-D114 (2012).

24. Ashburner, M. et al. Gene ontology: Tool for the unification of biology. The Gene Ontology Consortium. Nat. Genet. 25, 25-29 (2000).

25. Kohl, M., Wiese, S. \& Warscheid, B. Cytoscape: Software for visualization and analysis of biological networks. Methods Mol. Biol. 696, 291-303 (2011).

26. Liu, B. et al. Protective mechanism of 1-methylhydantoin against lung injury induced by paraquat poisoning. PLoS ONE 14, e222521 (2019).

27. R: A Language and Environment for Statistical Computing. \{R Core Team\}. R Foundation for Statistical Computing. (Vienna, Austria, 2020). https://www.R-project.org

28. Klop, B., Rego, A. T. \& Cabezas, M. C. Alcohol and plasma triglycerides. Curr. Opin. Lipidol. 24, 321-326 (2013).

29. Yamada, M. et al. Combined proteomic analysis of liver tissue and serum in chronically alcohol-fed rats. Alcohol Clin. Exp. Res. 37(Suppl 1), E79-E87 (2013).

30. Proietti, S. et al. Increase in motility and invasiveness of MCF7 cancer cells induced by nicotine is abolished by melatonin through inhibition of ERK phosphorylation. J. Pineal Res. 64, e12467 (2018).

31. Piubelli, C. et al. Proteomic changes in rat serum, polymorphonuclear and mononuclear leukocytes after chronic nicotine administration. Proteomics 5, 1382-1394 (2005).

32. Cecconi, D. et al. Serum proteomic analysis during nicotine self-administration, extinction and relapse in rats. Electrophoresis 29, 1525-1533 (2008).

33. Iwazaki, T., McGregor, I. S. \& Matsumoto, I. Protein expression profile in the striatum of acute methamphetamine-treated rats. Brain Res. 1097, 19-25 (2006).

34. Schindeler, A., McDonald, M. M., Bokko, P. \& Little, D. G. Bone remodeling during fracture repair: The cellular picture. Semin. Cell Dev. Biol. 19, 459-466 (2008).

35. Kushner, I., Edgington, T. S., Trimble, C., Liem, H. H. \& Muller-Eberhard, U. Plasma hemopexin homeostasis during the acute phase response. J. Lab. Clin. Med. 80, 18-25 (1972).

36. Kim, S. W. et al. Proteomics approach to identify serum biomarkers associated with the progression of diabetes in Korean patients with abdominal obesity. PLoS ONE 14, e222032 (2019).

37. Sheweita, S. A., Almasmari, A. A. \& El-Banna, S. G. Tramadol-induced hepato- and nephrotoxicity in rats: Role of Curcumin and Gallic acid as antioxidants. PLoS ONE 13, e202110 (2018).

38. Lin, W. H. et al. Seizure-induced 5-HT release and chronic impairment of serotonergic function in rats. Neurosci. Lett. 534, 1-6 (2013).

39. Rebholz, C. M. et al. Serum metabolomic profile of incident diabetes. Diabetologia 61, 1046-1054 (2018).

40. Leenaerts, D. et al. Plasma carboxypeptidase U (CPU, CPB2, TAFIa) generation during in vitro clot lysis and its interplay between coagulation and fibrinolysis. Thromb. Haemost. 117, 1498-1508 (2017).

41. Morser, J. et al. Carboxypeptidase B2 and N play different roles in regulation of activated complements C3a and C5a in mice. J. Thromb. Haemost. 16, 991-1002 (2018).

42. Tawara, S., Sakai, T. \& Matsuzaki, O. Anti-inflammatory and anti-fibrinolytic effects of thrombomodulin alfa through carboxypeptidase B2 in the presence of thrombin. Thromb. Res. 147, 72-79 (2016).

43. Sabbah, N., Jaisson, S., Garnotel, R., Angles-Cano, E. \& Gillery, P. Small size apolipoprotein(a) isoforms enhance inflammatory and proteolytic potential of collagen-primed monocytes. Lipids Health Dis. 18, 166 (2019).

\section{Acknowledgements}

We thank International Science Editing (http://www.internationalscienceediting.com) for editing this manuscript. We thank Shanghai Bioprofile Technology Co. Ltd. for supporting proteomics analysis.

\section{Author contributions}

Conceptualization: L.G. Data curation and formal analysis: S.J., G.L., H.Y. Investigation: E.X., W.X., X.Z. Methodology: W.X., X.Z. Supervision: L.G., J.L., G.L. Writing-original draft: S.J., L.G., G.L. Writing-review and editing: S.J., L.G. All authors reviewed the manuscript.

\section{Funding}

This study was funded by National Natural Science Foundation of China (81671864). 


\section{Competing interests}

The authors declare no competing interests.

\section{Additional information}

Supplementary Information The online version contains supplementary material available at https:/doi. org/10.1038/s41598-021-81109-7.

Correspondence and requests for materials should be addressed to L.G.

Reprints and permissions information is available at www.nature.com/reprints.

Publisher's note Springer Nature remains neutral with regard to jurisdictional claims in published maps and institutional affiliations.

(c) (1) Open Access This article is licensed under a Creative Commons Attribution 4.0 International License, which permits use, sharing, adaptation, distribution and reproduction in any medium or format, as long as you give appropriate credit to the original author(s) and the source, provide a link to the Creative Commons licence, and indicate if changes were made. The images or other third party material in this article are included in the article's Creative Commons licence, unless indicated otherwise in a credit line to the material. If material is not included in the article's Creative Commons licence and your intended use is not permitted by statutory regulation or exceeds the permitted use, you will need to obtain permission directly from the copyright holder. To view a copy of this licence, visit http://creativecommons.org/licenses/by/4.0/.

(C) The Author(s) 2021 